\title{
GLOBALIZACIÓN Y COMPETITIVIDAD: CONTEXTO DESAFIANTE PARA LA FORMACIÓN DE ENFERMERÍAa
}

\author{
Globalização e competitividade: contexto desafiante para a formação da enfermeira \\ Globalization and competitivity: challenging context to nurses' professional education
}

Vilma de Carvalho ${ }^{1}$

\begin{abstract}
RESUMEN
En esto texto se trata de contextualizar la globalización y competitividad en cuanto a la formación de enfermería. Son discutidos los aspectos de nuestro tiempo con sus crisis, cambios veloces, desequilibrios por las transformaciones sociales y avances científicos y tecnológicos. La utopía de una esencial unión mundial non asegura los derechos civiles para todos, y aún menos la idea de ciudadanía, en todas las partes, principalmente en relación a los profesionales de enfermería y los clientes o usuarios del sistema de salud. Se discute a propósito los asuntos de la enfermería y la formación de recursos humanos como parte de la crisis. El abordaje es de análisis-discursiva con destaque para la globalización qui non es ecuánime para todos en el mondo y para la competitividad un desafío permanente los trabajadores en el campo de la salud. La autora non presenta resultados investigativos, pero asume su posición en relación a la formación de enfermería, para la cual presenta un modelo curricular adoptado en el Brasil. Un modelo de formación coherente con la función de enfermeros para emprender los cambios en la práctica asistencial, para la formación del espirito científico y para la investigación consistente con la producción de resultados y construcción del conocimiento para el área de enfermería.
\end{abstract}

Palabras Clave: Enfermería. Educación en Enfermería . Habilitación Profesional

\section{Resumo}

Neste texto, trata-se de contextualizar globalização e competitividade em relação à formação profissional na enfermagem. São discutidos os aspectos de nosso tempo com suas crises, mudanças velozes, desequilibrios em vista das transformações sociais e dos avanços científicos e tecnológicos. A utopia de uma essencial união mundial não assegura os direitos civis para todos, e menos ainda a ideia de cidadania, em todas as partes do mundo, principalmente em relação aos profissionais de enfermagem e clientes ou usuários do sistema de saúde. A propósito, são discutidos os assuntos de enfermagem e a formação de recursos humanos como parte da crise de nosso tempo. A abordagem é analítico-discursiva com destaque para a globalização, que não é equânime para todos, e a competitividade como desafio permanente para todos os trabalhadores no campo da saúde. A autora não apresenta resultados investigativos, porém assume posição em relação à formação profissional na enfermagem, para a qual apresenta um modelo curricular adotado no Brasil. Um modelo de formação coerente com a função de enfermeiros para empreender mudanças na prática assistencial, para a formação do espirito científico e para a pesquisa consistente com a produção de resultados e a construção do conhecimento para a área da enfermagem.

Palavras-chave: Enfermagem. Educação em Enfermagem . Qualificação Profissional.

\section{Abstract}

This text contextualizes globalization and competitiveness in relation to nursing professional education. Present days aspects and their crisis, speedy changes, imbalances given social changes and scientific advances are discussed. The utopia of a core world unity does not grant equal civil rights for all, much less the citizenship idea all around the world, especially in relation to nursing professionals and clients or users of the health system. Nursing issues and human resources formation are discussed as part of present days' crisis. The approach is analytic-descriptive highlighting globalization, which is not equal for all, and competitiveness as a permanent challenge to all workers in the health area. The author does not present investigative results, however, takes a stand in relation to nursing professional education, to which presents a curricular framework or model adopted in Brazil. A framework model that is coherent with nurses' role in implementing changes in care practice, in developing scientific critical thinking and conducting research consistent with outcomes, and in knowledge building to nursing.

Keywords: Nursing. Education, Nursing. Credentialing.

${ }^{1}$ Enfermera (EEAN). Doctora/Docente Libre e Profesora Emérita (UFRJ). Profesora Titular de Enfermería de Salud Publica (DESP/EEAN). Bachiller y Licenciada en Filosofía (Facultad de Educación, UERJ). Líder de Grupo de la Línea de Investigación Para Una Epistemología de Enfermería (EEAN / CNPq). Active Member Sigma Theta Tau International Honor Society of Nursing / Rho Upsilon Chapter (USA). 
Globalización y competitividad: contexto desafinate para la Carvalho V

\section{SOBRE EL CONTEXTO EN GENERAL}

El tema que yo voy a tratar non es algo de inesperado. Los colegas de profesión, en Brasil, saben que por cerca de los últimos treinta años yo tengo por principal preocupación y actividad de labor en la educación universitaria, de hecho, la formación de enfermería. Pero, el contexto desafiante de la globalización y competitividad es ahora asunto imperioso, que acomete la atención y los intereses de todos en el mondo.

Non es tan simple contextualizar. Todos están atentos a las dos palabras globalización y competitividad. Ellas abarcan los eventos circundantes, acontecimientos mundiales y, quizás, casi todas las personas ya asumen la conciencia de una realidad de muchos desafíos por las transformaciones científicas, tecnológicas y sociales. Pero, cabe atención a las dos palabras, separadamente. Globalización es una palabra compleja y admite contradicciones. Es una palabra-llave para la coherencia con la realidad de cambios veloces de un mondo en crisis, que se quiere totalizado como se fuera un solo hogar para todos los hombres. Como la entiendo, competitividad es una palabra de consecuencia o de implicación. Su relevancia sucede en nivel de competición por la calidad de las acciones o actividades de pensar y hacer las cosas del humano trabajo en el plan de la propia globalización. La perspectiva, todavía, en el ámbito de la competitividad es que los trabajadores disputen sus posiciones través de los embates y disputas por la más plena calidad.

Sobre la palabra globalización, la utopía es la de colocarse todos los hombres bajo a una orden global, con el objetivo mayor de mantener asegurados los derechos de los ciudadanos civiles y, así, el mejor potencial equilibrio para todos. Pero un equilibrio que nadie comprende plenamente. Basta tener en la mente y percepción atenta que hay la marca de diferencias y riesgos en las situaciones humanas por todas las partes del mondo. Poca atención merece el caso de haber algunas personas que dominan sus privilegios o que otras sean como los carentes de toda la suerte. Non hay derechos asegurados para todos, en cuanto se habla de distribución de renta, trabajo, empleos, moradas, alimentos y vestuarios, la educación y salud, y poco o ninguna importancia se hay condiciones de libre transito de un hogar para otro. Lo más que se puede mirar, con efecto, es una realidad donde non hay estabilidad cierta para nadie, las oportunidades son desiguales, y hay violencias y contestaciones por todas las partes en el mondo.

Todos, o casi todos, son unánimes en cuanto hablan de la globalización en general, pero en el sentido estricto, la palabra es como un fenómeno controversial que se desarrolla por todos los países y regiones continentales. En realidad, la globalización manifestase con elementos non ecuánimes en todas las partes. Por veces son elementos distintivos, por veces son contradictorios y, así, se transmuta nuestro pensamiento, nuestro modo de hacer las cosas, nuestras vidas en la familia y lo que aprendemos a hacer con nosotros en las comunidades. La mutación es la tonalidad más constante. Por motivo de incesantes innovaciones producidas por la ciencia y tecnología, los cambios resultan en repercusiones esperadas o inesperadas. Y las transformaciones trazan determinantes y prioridades políticas, económicas y sociales. Algunos puedan suceder como ciertos, y otros non.

Con justa razón, casi nadie ignora más el sentido general de la palabra. Ahora los computadores están integrados en nuestro modo de vivir, permitiendo la idea de un contexto globalizado, nos cometiendo a todos y nos relacionando con lo que está más distanciado y también con el que está adelante de los propios ojos. La Internet, a su vez, hace sus milagros, casi se encuentra a la disposición de uno cualquier, desde que ya se puede conectar el computador con las personas en distantes países y variados hogares. Esto ha tornando posible que todos, o casi todos, pueden compartir las informaciones través de la telecomunicación, la cual se ha tornado una cuestión básica, fundamental para nuestro humano estilo de vivir. Todo el noticiario en el mondo puede ahora ser compartido con todos. El planeta tierra es como una gran morada. Las noticias avanzan veloces, gracia a la moderna telecomunicación. Basta que uno se disponga a usar el indicado aparato de telefonía celular o el personal notebook a su plena disposición, sea por el simple placer de integrar la totalidad mundial, o sea solamente porque está así ya determinado por las nuevas necesidades generadas por la globalización.

En consecuencia, todo se cambia al nuestro derredor y hasta mismo en la intimidad de nosotros. La velocidad de los cambios ha tornado la mutación la propia realidad, un fenómeno común de nuestra vida. El desafío mayor es la mutación en sí. Como todo se cambia, las situaciones humanas también. Todos los individuos aún que distintos, y con sus diferencias culturales en todas las regiones del mondo, son abarcados por los elementos distintivos de la onda globalizante. Sin embargo, ahora todos vivimos un período de crisis sin precedentes. En palabras de un importante autor britanico, el cual escribe sobre las crisis desafiantes de nuestro tiempo. ${ }^{1: 7}$

\section{(...) "a pesar del nuevo y de las innovaciones, - con todos los desafíos -, ahora vivimos un tiempo de ansiedad - o de agonía - porque las mismas cosas de antes ahora son diferentes; nuestro humano hábito ya non permanece como siempre, ... con esto, el futuro ya se adentra por la nuestra puerta, ... y los cambios no confieren más con nuestras cotidianas necesidades yopciones".}

Sin embargo, con la globalización non se trata solamente de tener todas las personas con derechos a la toda parafernalia del estilo tecnología digital de largo alcance y alta resoluciónTV de la tela-plasma, vídeos, diferentes aparatos de telefonía celular y computadoras particulares o personalizados. Sin 
embargo las señas personales non aseguran cualquier protección de privacidad. En la globalización, vivimos un tiempo dramático e de transición histórica sin cualquier antecedente. Los cambios y desafíos más evidentes producen otras tantas transformaciones, principalmente en las cuestiones de la educación y salud. Todo se cambia, en nuestras actitudes y comportamientos: - un nuevo pensar y modo de sentir influyen ahora nuestro estilo de ser, de tener, de existir. En las palabras de otro contemporáneo pensador británico ${ }^{2}$, en nuestro tiempo y en el mondo de hoy lo que sucede está ya determinado

(...) "por la globalización. Todavía, es como si el mondo estuviese en cierto desequilibrio o mismo en pleno descontrole; el mondo se queda demarcado por los cambios veloces, por muchos riesgos, por graves desafíos que alteran las cuestiones individuales y culturales. Ningún aspecto de la realidad escapa: - la tradición, la familia, las situaciones humanas en descompaso político-económico en todas las partes y, así, se transforma la sociedad misma" 2: 14-16.

Además, bajo la fuerza de la globalización, casi non hay más sigilo sobre las cuestiones de la vida. El consecuente desafío es saber (...) lo que la globalización está haciendo de nosotros (subtítulo de libro "Mundo en descontrole" 2). Entre los indígenas de la región amazónica de Brasil nadie ignora plenamente el sentido general de esta palabra. Y los indígenas saben que non es solo una cuestión de tener productos tecnológicos en sus manos 0 de tener derecho a la tierra adonde habitan. Así como saben manejar la TV, todos o casi todos ya saben como colocar y contraponer sus intereses particulares y contestar en provecto de sus derechos personales 0 grupales. Además ellos saben sobre el gran desafío de todos. Cualquier uno de lo liderazgo indígena es bien posicionado y sabe el modo de como poner adelante del interese personal el derecho tribal a la educación y salud. Todos saben lo más que se refiere a sus necesidades de obtener el apoyo o ayuda profesional de los recursos humanos adecuados y plenamente habilitados profesores, médicos y enfermeras.

En cuanto a la educación básica o universitaria, los brasileños como todos en América Latina saben de lo que necesitan de la educación y salud y saben de sus derechos, además, como todo ciudadano en el mondo de hoy. A pesar de las diferencias culturales, en cuanto a los objetivos del bien común, todos son unánimes, y asumen posición dispuestos a contestar por justicia en favor de lo que es el bien social. Y es donde entra el caso de la cuestión magna de la formación profesional y - en el caso de nuestro interese - la formación de enfermería.

\section{LAS LÍNEAS GENERALES DEL CUADRO SANITARIO Y EDUCACIONAL}

Para hablarse de la formación de enfermería non es preciso resaltar o apuntar, en esto texto, los datos estadísticos en detalles. En los países llamados emergentes, y mismo en otras partes del mondo, el cuadro sanitario en la primera década del siglo XXI es marcadamente de la morbilidad y la mortalidad alta, y hay un panorama de desequilibrio como que generalizado. A pesar de las diferencias culturales, hay un descontrole totalizado y más agravado por problemas generados por calamidades del ecosistema y por la violencia urbana y periférica. La asistencia a las personas enfermas o non enfermas, en cuanto sea conforme los planos del sistema de salud y bajo la política de gobiernos, non consigue dar la atención necesaria a todos los usuarios previstos por lo sistema político o por derecho civil. Hay problemas de asistencia por la insuficiente cantidad de los recursos humanos; hay dificultades por deficiencia material-instrumental para la sustentación de los mecanismos gerencial-administrativos de trabajo. Y hay serios desafíos por la baja extensión de la cobertura de salud para los individuos y comunidades que se quedan, al final, con deficiencias paralelas a la poca 0 ninguna atención de salud ya prevista en los planos de gobiernos, o quizás por las insuficientes providencias ya previstas en los planos de seguridad medico-social ${ }^{3}$.

Non parece necesario trazar el perfil epidemiológico de las populaciones por características específicas. En verdad, en cada país de la América Latina, los problemas y dificultades son propios del continuum salud-enfermedad. En esto momento, mezclado por muchas enfermedades, el cuadro sanitario se presenta con destaques aún para las enfermedades infectocontagiosas, algunas ya previstas, pero sin protocolos de atención, otras del estilo pandemias infecciosas inesperadas. La atención a la salud, mismo con el apoyo de las políticas de gobiernos, non consigue dar cuenta de una asistencia plenamente justa y necesaria para todos. Los riesgos de morir se encuentran ahora más relacionados a las enfermedades non previstas o non controladas.

A pesar de tentativas de ajustamientos en los planos del sistema de salud, en toda la América Latina, en todos los países, o casi todos, aún se observa los antiguos riesgos de muerte por enfermedades crónico-degenerativas, patologías de cáncer y de corazón, accidentes de tránsito y de trabajo. Aún son presentes los graves problemas de período grávido-puerperal de la mujer, con dificultades desde a la neo-natalidad mal asistida hasta los riesgos de tener sus hijos sin la debida o eficiente ayuda. Y ahora hay por toda parte el alto índice de personas en la tercera edad. Además, ningún de los países se ha libertado de los problemas de salud aliados a la precaria educación y, acrecidos, por las nocivas condiciones del insuficiente saneamiento básico y la pobreza en sí $^{3}$. 


\section{SOBRE LA FORMACIÓN PROFESIONAL Y LA COMPETITIVIDAD}

La educación profesional y la preparación de los recursos humanos para el campo de trabajo y para la vida es un asunto en constante cuestión. Mejor decir, consta en todas las agendas de discusión académica o de política gubernamental y, por cierto, abarca a todos los interesados - los profesionales en todas las áreas y la sociedad misma. Con efecto, la educación básica es una cuestión a espera de soluciones definitivas. Razón porque hay una mayor parte de la populación a la margen de los objetivos de lo que se entienda por educación de naturaleza y estructura continuada o permanente. El campo de la salud abarca quizás el área más procurada por sus mejores oportunidades. Todavía, el caso de la Enfermería, en el contexto de la globalización y competitividad, es una cuestión que requiere la urgencia de reconsiderarse la enseñanza y la formación de un perfil profesional adecuado a la necesidad de una firme posición frente a los desafíos de nuestro tiempo. Antes de todo, es preciso tener la humildad de una posición pedagógica de consenso para cambiar los objetivos, - casi siempre basados en la autoridad de los maestros -, por otros consistentes con la interrelación actuante de pareceros o compañeros solidarios. Lo imperativo non es más la búsqueda de respuestas, pero la procura permanente de las preguntas apropiadas a la duda científica y a los riesgos de perseguir lo que es problemático 0 incierto.

Lo más relevante ahora es el apoyo de una pedagogía de la incertidumbre ${ }^{4}$ o de lo cier to través de la inquisición u cuestionamiento de lo incierto. En la complejidad de las situaciones humanas y, así también, de las experiencias de la aprendizaje, es necesario el refinamiento pedagógico justo por el propósito de "una educación adecuada a la enfermería en nuestra época" 5:115. Una educación que pueda abarcar los asuntos de la enfermería - enseñanza y perfil profesional. De un punto de vista crítico, parece urgente la discusión de la temática con principios de pertinencia profesional, sea para el tiempo de hoy o para el futuro. Hay que tener en alta relevancia las cuestiones de la formación de enfermería, sobretodo, en la educación universitaria, pero también en la mira de un perfil de enfermero generalistay en cuanto a la capacitación para atender a todos los que demandan, en la práctica, los cuidados de enfermería. Con esto, son imperiosas las consecuentes implicaciones de aplicabilidad y de realización de currículos, programas y proyectos conjuntos de la educación y salud, con base en la investigación, para el enfrentamiento de los desafíos de hoy y del futuro.

El aspecto agudo de la cuestión es, precisamente, esto de aquilatarse un perfil profesional para los enfermeros para esta época de globalización. Un perfil que sea consistente non solo a la actuación en la práctica asistencial, principalmente que sea adecuado a la formación del espirito científico para los emprendedores de cambios, con atributos coherentes con las acciones de transformaciones sociales y la investigación. La más fuerte implicación, con justa razón, apunta a la competitividad. Un trazo del perfil profesional, más característico quizás, en razón de la necesidad de superar dominios non exclusivos del Saber de Enfermeríay de su conocimiento propio. Una tonalidad especíica en cuanto a la pertinencia de la práctica profesional y a la inherencia de los cuidados de enfermería. Hay que prepararse los estudiantes, prima facies hasta la última instancia, para la competitividad en su grado mayor de relevancia. Pues, los desafíos llegados con la globalización implican en asumirse las funciones de la Enfermería non solo para los enfrentamientos de la tecnología de punta o para las actividades de asistencia en las unidades de terapia intensiva. La preparación profesional indicada es para la formación de enfermería coherente con la asistencia en las comunidades. Y que sea de intención positiva para la cobertura de salud de la propia sociedad, como ya determinada por la idea de globalización.

Todavía, en Brasil, los mayores desafíos se quedan ahora aliados a la calidad de los atributos impuestos a las funciones de los enfermeros en los programas o proyectos de extensión clínico-asistencial o de salud de la familia. En realidad, en la América Latina, la intención de la política de salud ahora es de poner los profesionales de la educación y salud junto a los agrupamientos humanos $u$ organizaciones integradas a los sistemas de desarrollo industrial y social. El gran desafío está con la idea de poner los programas de educación y salud asociados a los programas de las empresas industriales, con la extensión universitaria asociada a los objetivos de gobiernos, y con intencionalidad objetiva a la atención para los grupos sociales. Poco o casi nada más interesa si la asistencia es en el área urbana o peri-urbana. Lo que interesa a la formación de enfermería,- - si es lo que más interesa a todos-, es el dominio de atributos coherentes a las estrategias para las funciones de los enfermeros en todos los sectores y hogares de la realidad de la salud, sea institucionalizada o no.

La peculiar función profesional es, sobretodo, de pertinencia de la Enfermería y puede ser refinada en las estrategias de la acción o interacción en los equipos interdisciplinarios. Mediante el propio modo de hacer las cosas en la práctica asistencial, las funciones de los enfermeros son compatibilizadas con la superación de los desafíos propios de la responsabilidad ética y social por los cuidados de enfermería. Con un aspecto destacado para los atributos sustantivos y adjetivos del papel profesional, esperase del enfermero, - como agente de una práctica social crítica-, que sea apto, también, para la competitividad en la practica de la investigación y producción de conocimientos científicos.

La educación y salud en plan internacional precisa, pues, ser coherente con los esfuerzos y objetivos de los que se dedican a la enseñanza y a la docencia universitaria en todos los países integrados por la política de la globalización. Estos esfuerzos 
son contribuyentes indispensables para mantenerse el equilibrio necesario a la educación y salud en cuanto

\section{(...) "a las directrices de una concepción comun o general de aprender-a-ser y de la búsqueda de perspectivas de tornarse un profesional de alta competencia - [o competitividad] - consistente con las nuevas direcciones del espirito contemporáneo frente al mondo que se hace y se desea totalizado frente a los derechos humanos. Solamente así, quizás, sea posible discutir todo lo más de interese de una política de la educación y salud y de lo humanismo científico del hombre y de la paz. Una concepción humanista, porque el hombre e sus realizaciones están en el centro; una concepción científica por el modo como el propio humanismo se queda definido y, así, más rico por las revoluciones resultantes de los contenidos de las ciencias" $": 25-26$.}

Además, es preciso tener en la conciencia que, actualmente, hay tendencia para la búsqueda de trabajo o de trabajadores para el ejercicio profesional fuera de sus países de origen. Non es preciso alargar la apreciación crítica. Pero ya es del conocimiento público la exploración del trabajo humano en el área de la salud y enfermería. Los jóvenes profesionales son invitados con promesas de buenos empleos y altos salarios $y$, después, son abandonados a su propia suerte en tierras distantes y, así, expuestos en las manos hasta de exploradores del trabajo en hogares domésticos o, quizás, con riesgos de se disponer a la pura y explorada actividad sexual. Sin la cuestión de la educación y salud en su mejor punto de equilibrio pedagógico en todo el mondo, no hay orden, non hay desarrollo, non hay humanismo y non hay paz. Y con eso, justo por implicación, hay que dedicarse esfuerzos a la formación de enfermería con posición decidida para haberse con las contingencias de un mondo que se propone como globalizado, pero con divergencias inaceptables de condiciones de vida y de trabajo. Si vivimos un tiempo de crisis, las consecuencias no son ecuánimes para todos. Las oportunidades de trabajo con estabilidad asegurada pueden hasta existir, pero son raras en la realidad de la educación y salud. Hay que tenerse en la mente que las transformaciones políticas, económicas, sociales están a imprimir otros cambios en los comportamientos éticos del hombre y justo, quizás, por la tendencia globalizante de suministrarse, hoy, hasta las necesidades básicas de los seres humanos.

Es necesaria una decisión ajustada en cuanto a la política en general y, en particular, sobre la política de la educación y salud. En el mondo globalizado - primera década del siglo XXI - las situaciones se traducen por un cuadro adonde se impera la orden de desorden. Una orden en desequilibrio o desorganizada por un trasgresormodo político-económico-social de ser, de tenery de existir. Si la orden es para todos, las reglas también. Todos quieren los mismos derechos y oportunidades en todo el mondo. La distinción puede venir con la capacitación para la competitividad, la cual cumple su papel o la función de un papel de imponer a todos el ideario ético de la calidad del ejercicio de las funciones profesionales. Todavía, para que así suceda, non se pueda dejar al lado los valores éticos y los predicamentos humanos, que más parecen en franca disolución. Todos que tratan de cuestiones de la enseñanza y de preparar los enfermeros para el trabajo en el campo de la salud, ya saben que es imperativa la practica de la investigación, sin la cual non hay solución de dificultades y desafíos. La práctica de la investigación es imperiosa en la conformación del perfil profesional, en cuanto adecuado a los tiempos de hoy y del futuro. Un perfil profesional competente para el enfrentamiento del desafío permanente por la búsqueda de empleo y trabajo y capaz de la positiva calidad de la acción en ámbito asistencial. Y, sobretodo, adonde los privilegios de la vida sean comprendidos en los significados de un mejor nivel de la felicidad del vivir para todos.

\section{ALGUNOS ASPECTOS DISTINTIVOS DE LA FORMACIÓN DE ENFEMERÍA}

A pesar de las diferencias culturales entre países y disparidades sociales entre las regiones internacionales, en respecto a la formación de enfermería, las situaciones humanas y los desafíos que yo tengo vivido en Brasil, así de momento, me parecen como se fueran situaciones de todos los tiempos de ayer, de hoy, quizá, de futuro. Sin embargo y mismo con los cambios veloces, las situaciones y experiencias que yo puedo transmitir son como permanentesy pueden interesar a todos.

La formación de enfermería, como la entiendo yo, por si, se refiere al interese de todas las sociedades en todas las regiones del mondo. En la América Latina, es fácil admitir que la cuestión es como pacífica. Las distinciones y diferencias no son grandes. Pues, todos los países latino-americanos siguen el paradigma de la Enfermería Moderna, instaurado hace ya casi 150 años, gracia a los esfuerzos y determinada misión de la gran fundadora Florence Nightingale. Mediante un plan de instrucción pedagógicamente adecuado, las nuevas Enfermeras Nightingale tuvieran su entrenamiento profesional en unidades anexas a Saint Thomas Hospital, en la ciudad de Londres, en el año de 1860 de la era de la Reina Victoria ${ }^{7}$.

Esto es, pues, un asunto de elevado sentido histórico y del interese mayor de todos los que viven la actividad académica de pensar la educación universitaria para formar enfermeros habilitados y proficientes. Sean ellos para integrar sus acciones en los equipos multidisciplinarios o interdisciplinarios, los enfermeros son profesionales de la salud, y son capaces de ofrecer su apoyo o ayuda espećfica y sus cuidados de pertinencia a todas las personas, familias y grupos de comunidades en 
cualquier parte del mondo. Ellos son competentes para los cuidados específicos en cantidad y calidad, como posibles y deseados por las personas frente a los desafíos de los desequilibrios del estado de salud. Y como la salud es un derecho universal para todos, por implicación, todos los clientes y usuarios del sistema de salud tienen el derecho a la asistencia de enfermería. El desafío principal, dentro de otros de la profesión, es la toma de posición en cuanto a la defensa de los derechos de los clientes - esta una dirección esencial del paradigma nightingaleano ${ }^{8}$. Y así, también, en cuanto al deber mayor de ofrecer los cuidados de enfermería adecuados al derecho de todas las personas de mantenerse en un nivel optimo de salud. Además, que sea esto, de hecho, el desafío mayor de hacer las cosas en el ámbito de la práctica asistencial de salud en nombre de la Enfermería.

En Brasil, - en la Escuela de Enfermería Anna Nery (EEAN/ UFRJ) -, desde el año de 1978 hemos obtenido el apoyo eficaz de lo Currículo de Nuevas Metodologías en nivel de Curso de Pregrado en Enfermería ${ }^{9}$. Gracia a un proyecto educacional con apoyo del Ministerio de la Educación, fueran alcanzados los cambios, objetivos, las metas y directrices del currículo. Los desafíos fueran muchos por los cambios educacionales o por la necesidad de formalizarse un nuevo perfil profesional para redefinirse la posición de la enfermera en la práctica. A la época, era urgente la introducción de la práctica de la investigación en la enseñanza, era imperativa la idea de una óptica más crítica sobre las acciones profesionales y la propia presencia de la enfermera en la asistencia de los clientes. Dos aspectos fueran destacados de pronto - el trabajo en la comunidady la idea de integración-y los dos aspectos ayudaran en la reorganización de las experiencias curriculares. El primero fue relevante para globalizar los conceptos y estrategias de hacer las cosas de la enfermería y de la práctica asistencial en hogares comprendidos como propios de la práctica, esta admitida en visión de conjunto, en el sentido de totalidad, sin la supremacía de unas partes sobre otras. Lo secundo aspecto, consistente a la integración de contenidos pedagógicos, y de las experiencias curriculares de los estudiantes.

Todos los esfuerzos fueran orientados por la idea de integración en tres sentidos - de la teoría a la práctica, del estudio al trabajo, y de la propia integración disciplinaria o de disciplinas. Pero, sin los esfuerzos eficientes de los profesores y, principalmente, sin la comprensión y concordancia de los estudiantes non teníamos alcanzado, quizás, el espacio para la realización de las directrices y objetivos o para obtener los cambios deseados.

En la mejor visión, los elementos pedagógicos de esto currículo se han extendido por todo el Brasil y quizás tenga alcanzado algunos países de América Latina. Con efecto, hemos tenido algunos estudiantes de Doctorado de otros países, realizando investigaciones comparativas de currículos, para evaluar el perfil del enfermero con formación universitaria o para aquilatar la función del enfermero en la práctica asistencial.
Non precisamos muchos detalles. El currículo fue idealizado para evitar la fragmentación de contenidos disciplinarios del proceso enseñar-aprender a ser un profesional de enfermería, sea para la acción en el mondo de hoy u sea para los enfrentamientos de desafíos del futuro.

Los cambios propiciaran la objetividad de la evaluación de los estudiantes, en cada etapa de la grade curricular, y con los avances de la aprendizaje interrelacionando la adecuación de los programas curriculares a la comprensión del sentido de la enfermería - lo que es y de lo que non es ${ }^{10}$. Principalmente, ahora todos los programas curriculares son de interese conjunto de todos los Departamentos de Enseñanza de la Escuela y, por eso, considerados InterDepartamentales.

Actualmente, la grade curricular pasa por algunos ajustes por fuerza de recientes directrices ministeriales y nuevos desafíos con la atención básica en general, y la salud de las familias y grupos comunitarios. Los mayores imperativos emergen consistentes en cuanto a la extensión universitaria para las comunidades al derredor de la Capital del Estado. En todos los programas curriculares, el foco central abarca las situaciones de los clientes, comprendidas como situacionesproblema no solo por las experiencias de la práctica asistencial variada, pero por la necesidad de la propia investigación. El abordaje preferencial es situacional- la propia situación es lo determinante para la acción - y en el ámbito del proceso de enfermería, se impone, también, el apoyo de la metodología de resolución de problemas ${ }^{11}$.

Las situaciones de interese de la enfermería abarcan los clientes que son todas las personas y grupos de la comunidad, sin cualquier distinción o exclusión; y como pueden estar situados en hogares institucionalizados o non ${ }^{12}$. Y, entonces, son las personas comprendidas 0 clasificadas como usuarios del sistema de salud, y como son los integrados de entidades educacionales regulares (profesores y estudiantes); los empleados de servicios públicos o privados con financiamiento de gobiernos (instituciones universitarias, hospitalarias, militares, empresas y fábricas); los asociados de organizaciones 0 entidades de amparo social o mantenidas con reglas y normas fundacionales o de custodia social; los trabajadores autónomos y otros desempleados o de trabajo libre de la economía precaria o flexible sin asignatura de servicio y registros de controle; las personas en tránsito (estaciones de viaje y de transporte interurbano); los individuos o familias sin casa y oportunidades de trabajo cierto y a la margen de la justicia social; y están incluidos los extranjeros independientemente de sus nacionalidades.

En las políticas de gobierno y del punto de una visión ampliada de la enfermería de salud pública como práctica social- en Brasil-la posición de enfermeros se ha firmado en torno de la idea de que 


\begin{abstract}
(...) "los clientes son todos los que se encuentran en cualquier fase del ciclo vital o del continuum salud-enfermedad; asípueden estar situados como previstos en los Programas de Asistencia Integral a la Salud: los adultos, las mujeres, el niño y los jóvenes, los trabajadores, las personas de tercera edady, además, todos los que sufren de limitaciones o deficiencias y que son carentes de procesos de rehabilitación física, psicológica y social" 12: 25-41.
\end{abstract}

Todavía, aún hay otros individuos que, - como los pobres de Dios -, viven a la margen de la justicia social 0 dependientes de la caridad de unos o de otros. Quizá, se quedan como mendigos, moradores de la calle, en su mayoría quizás viciados y, de toda forma, como receptáculos de todos los maleficios de las condiciones de la pobreza.

La viga maestra del currículo se apoya en el Marco Conceptual, una sintiese de pensar educacional para la formación de enfermería. Sin ser dependiente de la esquemática de programas de disciplinas variadas y separadas entre ellas, el currículo se realiza por los Programas Curriculares ÍnterDepartamentales (PCls). Todos asemejados en cuanto a la conformación estructural. Pero, distintos o ajustados por las situaciones de los clientes y, también, en cuanto a las experiencias y procedimientos del trabajo de cuidar en Enfermería. En conjunto, a pesar de los distintos clientes asistidos, el currículo través de los $\mathrm{PCls}$, conformase a un camino para la formalización del proceso enseñanza-aprendizaje, y como se fuera la marcha de los caminantes en la búsqueda de resolver las dificultades y problemas de los clientes. La misma andanza sirve para clarear el sentido esencial de la profesión de Enfermería. Con razón justa, las experiencias son seleccionadas para iluminar las cosas de la práctica y en cuanto sirven, también, para iluminar lo espirito de estudiantes y profesores. Como un todo organizado, través de los propios elementos básicos conceptuales y de experiencias, la integración manifestase en consolidación con el perfil profesional gracia a los elementos constitutivos del sentido horizontal y los que representan la propia acción en la verticalidad curricular.

En la práctica viva de la acción de enseñarse y de aprenderse, es la Enfermería misma que destacase en las actitudes y comportamientos que se formalizan para resaltar la nueva composición del perfil profesional deseado. Y, entonces, las metas y intereses curriculares suceden con base en el Marco Conceptual del Currículo de Nuevas Metodologías así descrito:

"La ENFERMERA actúa como el fulcro de un PROCESSO de lo cual emerge la práctica total de la ENFERMERÍA, entendida como la CIENCIA y la ARTE DEAYUDAR a los individuos, grupos y comunidades en SITUACIONES en las cuales non están capacitados par alcanzar su nivel óptimo de SALUD" 9:127.
Ese Marco efectiva su papel con palabras-llaves que sirven de énfasis a la idea y la perspectiva educacional en la formación de enfermería. Las palabras en letras capitales sirven a la formación del espirito crítico y a la comunicación de los significados principales de la relación enfermero-cliente, en teoría y estilo de la relación de ayuda y la totalidad de la práctica de enfermería ${ }^{13}$.

En la comprensión de las cosas en la totalidad de la práctica, el enfermero actúa como fulcro, porque es la base 0 cimiento y, al mismo tiempo, la palanca que equilibra y impele el movimiento de la acción. Pero, porque puede, también, generar y orientar los cambios en los rumos de la práctica y en el ritmo de la responsabilidad y obligación ética con el compromiso social. Además, el Marco es aún representativo de la concepción fundamental del pensamiento filosófico de la Escuela, y sirve de instrumento nuclear para retratar el enfermero en el ámbito de su acción frente a los intereses y desafíos de la práctica asistencial. Y, como una esencial, distintiva, dimensión sirve de punto de partida y punto de llegada para la arquitectura y desarrollo de todo el emprendimiento curricular.

\section{UNAS PALABRAS A MÁS PARA CONCLUIR}

De una óptica crítica, el proceso pedagógico en el área de enfermería, ya avanza como intermedio de una crisis a invocar nuevos ajustes y reconsideraciones de todos los elementos curriculares en nivel de Pregrado. Basta una atención especial a los trazos esenciales del perfil profesional. El enfermero en cualquier área de actuación practica precisa de atención constante para el significado de su trabajo y de sus funciones en cuanto al principio de la realidad misma. En esto sentido, precisamos de acuerdos sobre todos los intereses y significados de la Enfermería - ciencia y arte de ofrecer los cuidados de enfermería. Los clientes precisan de ayuda y defensa de sus derechos de usuarios de un sistema de salud precario, en gran parte injusto por la deficiencia de los recursos humanos de salud - no solo en nivel local, más también mundial. Y non se puede dejar de lado los intereses de la propia área de Enfermería y de su sentido como profesión. Al final, los clientes son asistidos, principalmente, por los cuidados de enfermería que, además, son evaluados como la ayuda prestada. Estudiantes y profesores, conjuntamente, son comprometidos con la evaluación de las experiencias, de los objetivos y de las metas del plan de la educación y salud y, sobretodo, por el propio sentido de la Enfermería.

En verdad, los contenidos disciplinarios constan de los programas y proyectos del plan global de enseñanzaaprendizaje. Pero, de toda forma, las experiencias con los clientes son determinantes para el aporte teórico-y esto es imperativo en nivel de Curso de Pregrado. Si las nuevas metodologías son adecuadas a la formación de profesionales de salud con perfil de enfermero generalista, entonces hay que 
Globalización y competitividad: contexto desafinate para la

tenerse la base educacional adecuada a la capacitación de refinamiento profesional continuado. Pero es preciso formar la mentalidad del enfermero consistente a un nivel de competencia pluridimensional. De todas las formas, el desafío educacional es la formación de enfermería con objetivos de formar el profesional en cuanto a las posibilidades de atención a los reclamos sociales. Y con vistas a la meta de alcanzar el dominio de conocimiento coherente con la resolución de las cuestiones de la salud y, principalmente, visando el ámbito de la área de Enfermería.

Non por la razón obvia, pero yo, personalmente, creo que la política de globalización y competitividad, por si sola, non hay de hacer milagros en cuanto a un contexto político con potencial capacidad para ofrecer el apoyo y sustentación a la idea de la formación de profesionales de la área de salud. Mismo que el paradigma nightingaleano se ha, finalmente, adaptado a la formación de la enfermería, en la realidad de los países de la Unión Europea, y así incluidos los países de la península ibérica - España y Portugal - non lo creo en soluciones ya definidas, o como se dije con las cartas colocadas en la mesa. Mucho más, creo que estamos ahora con carencia de consenso o de acuerdos sobre las directrices de política filosófica, de metas de la educación y salud, y de objetivos capaces de consubstanciar lo interese profesional para la propia Enfermería rumo al futuro.

Es necesario que la discusión de nuestras cosas de interese profesional tengan un sentido más universal para alargar la visión del mundo globalizado y comprender mejor los intereses comunes de todos, en todas las regiones del planeta. Ahora que nos encontramos todos viviendo los tiempos del Tercero Milenio, en medio a muchos cambios y transformaciones, quizás sea aún preciso ajustes y adaptaciones de programas de la educación y salud para atender a todos los más interesados - los asistidos y los asistentes. Pues, la globalización puede ser para lo que es bueno y, quizás, para lo que non es. Como la entiendo, pienso que la formación de enfermería debe adaptarse a las cuestiones profesionales y sociales en cada país, o por lo menos en cada región continental. Por lo más que non se quiera, las cuestiones de la educación y salud se resaltan con sus diferencias en toda parte. Todavía, a pesar de las diferencias culturales, los desafíos están presentes y se desarrollan través de las crisis de nuestro tiempo. El área de Enfermería, por implicación, non escapa de estar situada. Como tengo observado, justo por la tendencia de la onda de globalización, el asunto se presenta con más desafíos para las Escuelas y Facultades de Enfermería en todas, o casi todas, las Universidades en varias partes del mondo.

Los que son atentos a los acontecimientos circundantes, y que asumen su mejor posición en respecto a la conciencia de ser, de tenery de existir, ya saben que el mayor desafío nos congrega al encuentro del futuro. Un futuro condicional a las metas de las ciencias de las evidencias positivas, pero que nos remeta para el camino de las galaxias. El paradigma de las ciencias y sus tecnologías está, también, en cambio y con la fuerte pretensión de alcanzar el dominio de la realidad través de un holograma. El liderazgo en la área de las ciencias de la física ya piensa en como las cosas pueden ser convenientes para todos, en todas las partes. Esto, se es posible la utopía del alcance de una plena unión del planeta, y con organización coordenada por elementos de una nueva política de globalización y competitividad.

En consecuencia, los desafíos para asistir a los clientes que demandan nuestro trabajo y los cuidados de enfermería quizás sean realizados de otro modo. Los planes de educación y salud, en el futuro, ciertamente nos van imponer opciones condicionales y otras aptitudes frente a nuevos desafíos que no imaginamos y quizás tengamos de habernos con nuevas reglas de vivir y convivir. Non solamente los enfermeros, creo que todas las personas van precisar repensar sus intereses y su propia vida frente a nuevas formas de conjunción global de propósitos, metas y de objetivos para tener u suministrar todos los hombres en todas las regiones del mondo o, mejor decir, en todas las regiones del planeta.

La pregunta crucial que se coloca es: - ¿Quién sabe de todo el futuro? De mi propia posición, yo puedo afirmar - hay que preguntar hasta a lo propio Dios. Todavía, en cuanto esperase la divina respuesta, el potencial futuro de la formación de enfermería en el contexto de la globalización y competitividad bien que puede servir de pauta para muchas, sucesivas discusiones, pues el futuro ciertamente será fantástico.

\section{NOTA}

a Texto presentado en la X Conferencia Iberoamericana de Educación en Enfermería / II Congreso Latinoamericano-Europeo y III Seminario de Investigación de Educación en Enfermería de ALADEFE/UDUAL. Ciudad de Panamá, 26 - 30 de octubre de 2009.

\section{REFERENCIAS}

1. Toynbee A. 0 desafio de nosso tempo.Tradução de Edmond Jorge. Rio de Janeiro(RJ): Zahar; 1975.

2. Giddens A. Mundo em descontrole: o que a globalização está fazendo de nós. $3^{\mathrm{a}}$ ed. Tradução de Maria Luiza X. de A. Borges. Rio de Janeiro(RJ): Record; 2003. Proibida a venda desta edição em Portugal e resto da Europa

3. Fundación W. K. Kellogg Report. Enfermería del siglo XXI en América Latina $1^{\text {a }}$. ed sept. 1991. New York (USA): National League for Nursing; 1991.

4. Japiassu H. Pedagogia da incerteza e outros estudos. Rio de Janeiro(R)): Imago; 1983. 
5. Carvalho V, organizadora. Acerca de uma educação apropriada à enfermagem em nossa época. In: Sobre enfermagem: ensino e perfil profissional $1^{\mathrm{a}}$ ed. Prefácio de Lygia Paim. Rio de Janeiro (RJ): EEAN/UFRJ; 2006. p.115-34.

6. Faure E. Aprender a ser. Tradução de Maria Helena Cavaco e Natércia Paiva Lomba. São Paulo (SP): UNESCO/Difusão; 1972.

7. Dolan JA. Nursing in society: a historical perspective. $13^{\mathrm{a}}$ ed. Philadelphia/ London/Toronto: W.B.Saunders Company; 1973.

8. Seymer LR. Florence Nightingale: pioneira da enfermagem e precursora da emancipação feminina. Tradução de J. Guinsburg. São Paulo(SP): Melhoramentos; [19...]

9. Carvalho V, Castro IB, Paixão SS. Um projeto de mudança curricular no ensino de enfermagem em nível de graduação que favorece aos propósitos emergentes da prática profissional. Anais do $30^{\circ}$ Congresso Brasileiro de Enfermagem; 1978 jul. 16 - 22; Belém (PA), Brasil. Brasília (DF): ABEn; 1978.

10. Nightingale F. Notas sobre enfermagem: o que é e o que não é. Prefácio de leda Barreira e Castro. Tradução de Amália Correa de Carvalho. São Paulo (SP): Cortez Ribeirão Preto( SP): ABEn/CEPEn; 1989.

11. Abdellah FG, Beland IL, Martin A, Matheney. Patient-centered approaches to nursing $2^{\text {nd }}$ print. New York (USA): Macmillan; 1961.

12. Carvalho V. A enfermagem de saúde pública como prática social: um ponto de vista crítico sobre a formação da enfermeira em nível de graduação. Esc Anna Nery. 1997 jul; ano I (n esp lanç): 25- 41.

13. Carvalho V. A relação de ajuda e a totalidade da prática da enfermagem. Anais do $32^{\circ}$ Congresso Brasileiro de Enfermagem 1980 jun 1-7. Brasília (DF), Brasil. Brasília (DF): ABEn; 1980. p. 65 - 72. 